\title{
Lugol chromoendoscopic screening for esophageal dysplasia/early squamous cell carcinoma in patients with esophageal symptoms in low-risk region in China
}

\author{
YING SHAO ${ }^{1}$, ZHONG-LIN YU ${ }^{2}$, MING JI ${ }^{2}$, YONG-DONG WU ${ }^{2}$, YONG-ZHENG YU ${ }^{2}$, \\ XIAO-MEI LIANG ${ }^{2}$, LI YU ${ }^{2}$, YING-LIN NIU ${ }^{2},{\text { PENG } \text { LI }^{2} \text { and SHU-TIAN ZHANG }}^{2}$ \\ Departments of ${ }^{1}$ Geriatrics and ${ }^{2}$ Gastroenterology, Beijing Friendship Hospital, \\ Capital Medical University, Beijing 100050, P.R. China
}

Received June 11, 2014; Accepted February 24, 2015

DOI: $10.3892 / 01.2015 .3230$

\begin{abstract}
Detection of esophageal dysplasia/early esophageal squamous cell carcinoma (ESCC) is essential for improving 5 -year survival. The aim of this prospective study was to evaluate whether Lugol chromoendoscopy improves the detection of esophageal dysplasia/early ESCC in patients with esophageal symptoms in a low-incidence region in China. Eligible patients were randomly assigned into two groups who received routine endoscopy or Lugol chromoendoscopy. During endoscopy, between one and five biopsies were taken from visible lesions for routine endoscopy, or unstained areas of $>0.5 \mathrm{~cm}$ in diameter for Lugol chromoendoscopy. In total, 812 patients were enrolled, 395 for routine endoscopy and 417 for Lugol chromoendoscopy. The overall detection rate of esophageal dysplasia/early ESCC was $10.6 \%$ (86/812), the detection rates were 7.3\% (29/395) and $13.7 \%$ (57/417) in routine and chromoendoscopy groups, respectively $\left(\chi^{2}=8.58, \mathrm{P}=0.003\right)$. The detection rates were $8.3 \%$ (48/580), $17.2 \%$ (17/99) and $16.5 \%$ (22/133), respectively, in patients with reflux, dysphagia and globus sensation symptoms. In the chromoendoscopy group, 213 patients had unstained lesions of $>0.5 \mathrm{~cm}$, the detection rates of dysplasia/early carcinoma were $5.3 \%(4 / 76)$ in those with lesions of $0.5-1.0 \mathrm{~cm}$, and $37.2 \%(51 / 137)$ in those with lesions $>1.0 \mathrm{~cm}\left(\chi^{2}=21.46\right.$, $\mathrm{P}<0.001)$. These results indicate that Lugol chromoendoscopy improves the detection rate of esophageal dysplasia/early carcinoma in patients with esophageal symptoms compared with routine endoscopy. We propose that Lugol chromoendoscopy must therefore be considered in addition to routine endoscopy in patients with esophageal symptoms.
\end{abstract}

Correspondence to: Dr Shu-Tian Zhang, Department of Gastroenterology, Beijing Friendship Hospital, Capital Medical University, 95 Yong'an Road, Beijing 100050, P.R. China

E-mail: shutianzhangen@163.com

Key words: dysplasia, early esoghageal squamous cell carcinoma, lugol chromoendoscopy

\section{Introduction}

Esophageal carcinoma is the eighth most common cancer worldwide, responsible for 481,600 new cases in 2008, and the sixth most common cause of mortality from cancer with 406,500 deaths (1). Geographic variation in incidence is extremely striking, with China being high-risk and western Africa being low-risk. In China, 210,000 patients succumb to esophageal squamous cell carcinoma (ESCC) per year, accounting for $52 \%$ of the worldwide mortality due to ESCC (1). Although various treatments, such as surgery with preoperative treatments of radiotherapy, chemotherapy and hyperthermia, have been used, esophageal carcinoma has the poorest prognosis among digestive tract malignancies and the 5-year survival rate of advanced esophageal carcinoma is $<25 \%(2,3)$. However, the 5 -year survival rate of early esophageal carcinoma can reach $86-100 \%(4,5)$. Thus, early detection of ESCC appears to be the most promising method of improving the 5-year survival.

In recent decades, esophageal Lugol chromoendoscopy has become a common clinical approach in China, particularly in high-risk regions. Lugol chromoendoscopy has been demonstrated to improve endoscopic visualization of squamous dysplasia and carcinoma of the esophagus in Linxian, a high-risk ESCC region in the Henan Province in China (6). However, the diagnostic value of Lugol chromoendoscopy in low-risk regions is unknown. Beijing is a low-risk ESCC region; the morbidity $(9.2 / 100,000)$ and mortality $(7.5 / 100,000)$ is lower than the average level in China $(19.3 / 100,000$ and $15.4 / 100,000)$ and is similar to the global average level $(1,7)$. Our previous study demonstrated that Lugol chromoendoscopy elevates the detection rate of early ESCC/dysplasia in high-risk individuals in Beijing (8); additionally, patients with esophageal symptoms, such as reflux, dysphagia, and globus sensation, were often observed to have esophageal dysplasia or early esophageal carcinoma. It has been previously reported that $61.9 \%$ patients with early stage esophageal carcinoma exhibit subjective symptoms (9). Therefore, the aim of the present study was to conduct a prospective study to evaluate whether Lugol chromoendoscopy improves the detection of squamous dysplasia/early carcinoma of the esophagus in patients with esophageal symptoms in Beijing, China. 


\section{Materials and methods}

Subjects. The study was carried out at the Endoscopic Center of Beijing Friendship Hospital, Capital Medical University (Beijing, China). Patients with reflux, dysphagia, and globus sensation were included consecutively between January 2002 and May 2004, with the exception of the following: i) Patient had thyroid disease; ii) was hypersensitive to iodine; iii) was pregnant or lactating; iv) had chronic nephropathy; v) had esophageal varices, polypus, ulcer, hiatus hernia, diverticulum, and leiomyoma; vi) had any malignancies including esophageal advanced carcinoma; vii) lived in Beijing $<10$-years; or viii) had coagulation defects.

Patients were randomly divided into routine endoscopy and chromoendoscopy groups according to odd or even numbers prior to endoscopy examinations. Patients in the routine endoscopy group only received routine endoscopy examinations; patients in the chromoendoscopy group received routine endoscopy examinations, followed by iodine staining. At recruitment, each subject was interviewed to collect detailed information with regard to their history of alcohol and tobacco use. Alcohol use was defined as over $100 \mathrm{~g}$ per week over one year in this study. Light or heavy tobacco use were categorized by the approximate 50th percentile pack-years value among patients (as our previous study) (10); i.e. $<25$ or 25 pack-years [(cigarettes per day/20)x(years smoked)]. This study was conducted in accordance with the declaration of Helsinki. This study was conducted with approval from the Ethics Committee of Beijing Friendship Hospital, Capital Medical University. Written informed consent was obtained from all participants.

Endoscopic procedures. All subjects were given $5 \mathrm{ml}$ of $1 \%$ lidocaine for local anesthesia 2-5 min prior to endoscopy. Endoscopic examination was performed by an experienced endoscopist using a videoendoscope (GIF-XQ240; Olympus Optical Co., Ltd, Tokyo, Japan). In the routine endoscopy group, the entire esophagus and upper gastrointestine was examined and between one and five biopsies were collected for pathology from any areas with abnormal lesions (Table I) (7). In the chromoendoscopy group, following routine endoscopic examination, the tip of the endoscope was placed in the distal part of the esophagus and $10 \mathrm{ml}$ of $2.5 \%$ Lugol's solution $(25 \mathrm{~g} \mathrm{I}+50 \mathrm{~g} \mathrm{KI}$ in $1000 \mathrm{ml}$ distilled water; $2.5 \%$ weight/weight iodine) was sprayed onto the esophageal surface from the gastroesophageal junction to the upper esophageal sphincter using the dye-spraying catheter (PW-5L-1, Olympus Optical Co., Ltd) through the biopsy channel. From each unstained or light-stained area of $>0.5 \mathrm{~cm}$ in diameter, between one and five biopsies were collected for pathology. All characteristics, size and location in visually abnormal areas or unstained areas in the chromoendoscopy group were described and photographed.

Histological examination. The biopsies were fixed in $10 \%$ formalin, embedded in paraffin, cut into $5 \mu \mathrm{m}$ sections, and examined after staining with hematoxylin and eosin. An experienced pathologist analyzed the histology samples. Dysplasia was defined as an atypia of the epithelium with a basophilic matrix, a high matrix-core ratio, and a hyperheterochromatin. It was histologically classified into three grades: Mild (abnormalities confined to the lower one-third of the epithelium); moderate (abnormalities in the lower two-thirds); and severe grade (immature cells occupied more than two-thirds of the epithelium), according to the degree of atypical epithelium in comparison with the basal zone (11). Early esophageal carcinoma was defined as a tumor not beyond mucosal $2(11,12)$. When several lesions were observed in a patient, only the most severe lesion was considered.

Statistical analysis. Numerical data is presented as the mean \pm standard deviation (SD) and categorical data is expressed as percentage. Difference was evaluated by AVOVA, $t$-test or $\chi^{2}$ tests. $\mathrm{P}<0.05$ was considered to indicate a statistically significant difference. All tests were two-sided tests and all analyses were conducted using Statistical Analysis System Software (version 6.12, SAS Institute, Cary, NC, USA).

\section{Results}

General information. In total, 900 patients were randomized to receive routine endoscopy (450 patients) and chromoendoscopy (450 patients) examinations. Due to polypus, ulcer, hiatus hernia, diverticulum, or leiomyoma, 55 and 33 patients were eliminated in the routine endoscopy and chromoendoscopy group, respectively. A total of 812 patients who fulfilled the inclusion and exclusion criteria were enrolled. The baseline characteristics of the 395 patients in the routine endoscopy group and 417 patients in the chromoendoscopy group are summarized in Table II. The patients in the chromoendoscopy group and the control group were comparable in age, gender, symptoms and smoking and alcohol status. The mean age was $57.08 \pm 12.43$ years for the chromoendoscopy group and $55.66 \pm 13.30$ years for the control group $(\mathrm{P}=0.12)$. The number of patients $>45$ years of age for the two groups was determined, and no significant difference was identified (86.6 vs. $75.2 \% ; \mathrm{P}=0.12$ ). Additionally, no significant difference was identified in gender distribution $(\mathrm{P}=0.89)$, smoking and alcohol status $(\mathrm{P}=0.53)$, and symptoms $(\mathrm{P}=0.27)$.

Detection of dysplasialearly carcinoma. A total of 86 patients had dysplasia/early carcinoma, and the detection rate was $10.6 \%$ (86/812). In the routine endoscopy group, 29 patients $(7.3 \%, 29 / 395)$ had dysplasia/early ESCC. The detection rates of mild, moderate and severe dysplasia/early ESCC in the routine endoscopy group were 3.3\% (13/395), 2.8\% (11/395) and $1.3 \%(5 / 395)$, respectively. In the chromoendoscopy group, $57(13.7 \%, 57 / 417)$ dysplasia/early carcinoma patients were identified, among whom, $31(7.4 \%, 31 / 417)$ patients exhibited mild dysplasia, $12(2.9 \%, 12 / 417)$ patients had moderate dysplasia and $14(3.4 \%, 14 / 417)$ patients exhibited severe dysplasia/early ESCC. A significant difference was observed in the detection rates of dysplasia/early ESCC between the two groups $\left(\chi^{2}=8.58, \mathrm{P}=0.003\right.$; Table III). Among the 57 cases of dysplasia/early ESCC in chromoendoscopy group, 18 cases of esophagitis were revealed endoscopically; mild, moderate and severe dysplasia were present in 11,6 and 1 cases, respectively. In the chromoendoscopy group, the most common appearance of severe dysplasia/early ESCC (64.3\%, 9/14) was rugged or rough surface under routine endoscopy, also showing irregular unstained areas after staining with Lugol's solution (100\%, 14/14; Fig. 1). 
Table I. Abnormal lesions of esophagus under routine endoscopy.

\begin{tabular}{ll}
\hline Abnormal lesions & \multicolumn{1}{c}{ Characteristics } \\
\hline $\begin{array}{l}\text { Irregular } \\
\text { Small white patch }\end{array}$ & $\begin{array}{l}\text { Mucosa was focally or diffusely irregular, with prominent wrinkling } \\
\text { Focal raised or flat white patch, with smooth distinct borders, usually }<1 \mathrm{~cm} \text { in greatest } \\
\text { dimension }\end{array}$ \\
Focal red area & $\begin{array}{l}\text { Focal flat red area not caused by mucosal contact } \\
\text { Erosion }\end{array}$ \\
Focal defect in the mucosa, the erosions were subcatergorized as linear, punched-out, \\
or broad-based, depending on their shape and size \\
Mucosa was focally thickened and raise, with irregular indistinct borders and occasional \\
shallow surface erosions, usually were $>1$ cm in greatest dimension
\end{tabular}

Table II. Baseline characteristics of demographic, smoking, alcohol status and symptoms between the routine endoscopy and chromoendoscopy groups.

\begin{tabular}{|c|c|c|c|c|c|}
\hline \multirow[b]{2}{*}{ Variable } & \multicolumn{2}{|c|}{ Chromoendoscopy $(n=417)$} & \multicolumn{2}{|c|}{ Routine endoscopy $(n=395)$} & \multirow[b]{2}{*}{$\mathrm{P}^{\mathrm{a}}$} \\
\hline & $\mathrm{n}$ & $\%$ & $\mathrm{n}$ & $\%$ & \\
\hline Gender & & & & & 0.89 \\
\hline Male & 243 & 58.3 & 232 & 58.7 & \\
\hline Female & 174 & 41.7 & 163 & 41.3 & \\
\hline Age structure & & & & & 0.12 \\
\hline$\geq 45$ & 362 & 86.8 & 297 & 75.2 & \\
\hline$<45$ & 55 & 13.2 & 98 & 24.8 & \\
\hline Smoking status & & & & & 0.90 \\
\hline Never & 331 & 79.4 & 315 & 79.7 & \\
\hline Ever & 86 & 20.6 & 80 & 20.3 & \\
\hline$<25$ pack-year & 46 & 53.5 & 36 & 45.0 & 0.27 \\
\hline$\geq 25$ pack-year & 40 & 46.5 & 44 & 55.0 & \\
\hline Alcohol status & & & & & 0.53 \\
\hline Yes & 48 & 11.5 & 40 & 10.0 & \\
\hline No & 369 & 88.5 & 355 & 90.0 & \\
\hline Symptoms & & & & & 0.27 \\
\hline Reflux & 272 & 68.9 & 308 & 73.9 & \\
\hline Dysphagia & 54 & 13.7 & 45 & 10.8 & \\
\hline Globus sensation & 69 & 17.5 & 64 & 15.3 & \\
\hline
\end{tabular}

${ }^{\mathrm{a}} \mathrm{P}<0.05$ compared between groups.

Association between the dysplasialearly ESCC and esophageal symptoms. Patients were divided into three groups according to symptoms. Overall, 580 cases had reflux, 99 cases had dysphagia, and 133 cases had globus sensation. The detection of mild, moderate and severe grade dysplasia/early ESCC in the three groups was $8.3 \%$ (48/580), $17.2 \%$ (17/99), and $16.5 \%$ (22/133), respectively (Table IV).

Association between the detection rates and the length of unstained lesions (USLs). USLs were observed in 213 patients $(>0.5 \mathrm{~cm})$. The patients were divided into two groups according to the length of USLs. If one patient had more than one USL, the largest lesion was selected to group. In total, 76 cases had
USLs of $0.5-1.0 \mathrm{~cm}$ in diameter. Among these cases, 4 patients had dysplasia/early ESCC (4 mild dysplasia cases). USLs of $>1.0 \mathrm{~cm}$ were observed in 137 seven cases and the number of mild, moderate and severe grade dysplasia/early ESCC was 27, 10 and 14, respectively. A significant difference was identified between the two groups $\left(\chi^{2}=21.46, \mathrm{P}<0.001\right.$; Table V).

\section{Discussion}

Lugol chromoendoscopy is an important diagnostic tool and has been widely used to detect esophageal carcinomas or precancerous lesions as it can make the borders of visible lesions more clear or reveal lesions that cannot be observed by 
Table III. Detection rates of mild, moderate and severe grade dysplasia/early ESCC.

\begin{tabular}{lcccccc}
\hline \multirow{2}{*}{ Groups } & & & \multicolumn{2}{c}{ Dysplasia/early carcinoma (\%) } & & \\
\cline { 4 - 5 } & No & Mild & Moderate & Severe/early ESCC & Total & Others ${ }^{\mathrm{a}}(\%)$ \\
\hline Chromoendoscopy & 417 & $31(7.4)$ & $12(2.9)$ & $14(3.4)$ & $57(13.7)$ & $360(86.3)$ \\
Routine endoscopy & 395 & $13(3.3)$ & $11(2.8)$ & $5(1.3)$ & $29(7.3)$ & $366(92.7)$ \\
$\mathrm{P}^{\mathrm{b}}$ & & 0.009 & 0.936 & 0.049 & 0.003 & \\
\hline
\end{tabular}

ancluding normal (228 vs. 244 cases), inflammation (102 vs. 107 cases), and benign hyperplasia (22 vs. 23 cases) (Chromoendoscopy vs. Routine endoscopy). ${ }^{\mathrm{b}}$ Two sided $\chi^{2}$-test.

Table IV. Association between the detection rates of dysplasia/early ESCC and esophageal symptoms.

\begin{tabular}{lcccc}
\hline Histological findings & Reflux $(\%)$ & Dysphagia $(\%)$ & Globus sensation (\%) & Total (\%) \\
\hline Dysplasia/early ESCC & $48(8.3)$ & $17(17.2)$ & $22(16.5)$ & $86(10.6)$ \\
Mild & $28(4.8)$ & $7(0.07)$ & $10(7.5)$ & $44(5.4)$ \\
Moderate & $9(1.6)$ & $6(0.06)$ & $8(0.06)$ & $23(2.8)$ \\
Severe/early ESCC & $11(1.9)$ & $4(0.04)$ & $4(0.03)$ & $19(2.3)$ \\
Others & $532(91.7)$ & $82(82.8)$ & $112(84.2)$ & $726(89.4)$ \\
Total & $580(71.4)$ & $99(12.2)$ & $133(16.4)$ & 812 \\
\hline
\end{tabular}

Table V. Association between the detection rates and the length of unstained lesions in chromoendoscopy group.

\begin{tabular}{lccccc}
\hline & & \multicolumn{4}{c}{ Dysplasia (\%) } \\
\cline { 3 - 5 } Groups & No & Mild & Moderate & Severe/early carcinoma & Total \\
\hline $0.5-1.0 \mathrm{~cm}$ & 76 & $4(5.3)$ & $0(0)$ & $0(0)$ & $4(5.3)$ \\
$\geq 1.0 \mathrm{~cm}$ & 137 & $27(19.7)$ & $10(7.3)$ & $14(10.2)$ & $51(37.2)$ \\
$\mathrm{P}$ & & 0.04 & 0.22 & 0.04 & $<0.001$ \\
\hline
\end{tabular}
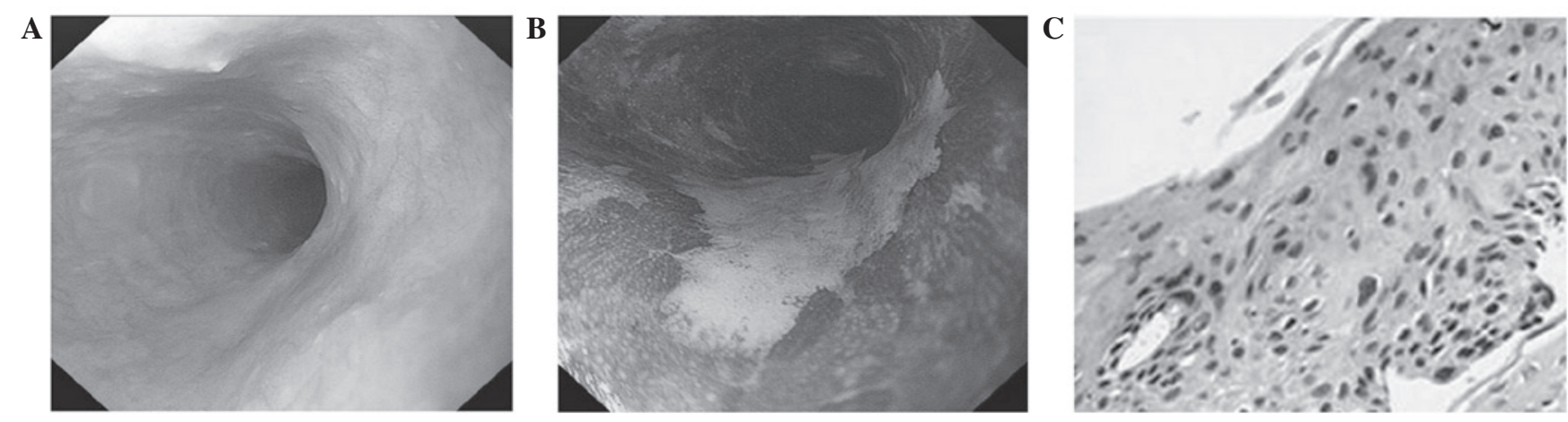

Figure 1. The view of early ESCC under routine endoscopy and chromoendoscopy and pathological changes. (A) Routine endoscopic view of esophagus with rough surface. (B) Chromoendoscopic view of esophagus with 2.5\% Lugol's solution staining showed irregular unstained areas. (C) Histologic examination of unstained area showed immature cells occupying more than three quadrants of the epithelium (hematoxylin and eosin staining; magnification, $\mathrm{x} 200)$.

routine endoscopy examinations. It was reported that $27 \%$ of moderate/severe dysplasia of the esophagus were not identified in routine endoscopy examinations (6). In previous studies, Lugol chromoendoscopy was predominantly used in high-risk populations or high-risk regions to screen esophageal carcinomas or precancerous lesions $(6,13-15)$. The current study screens esophageal dysplasia/early carcinoma in low-risk regions using Lugol chromoendoscopy to evaluate whether 
this technology may improve the detection rates of esophageal dysplasia/early carcinoma. The results revealed that the detection rate of dysplasia/early carcinoma in chromoendoscopy group was markedly higher than that of the routine endoscopy group. The result suggested that Lugol chromoendoscopy was also important in low-risk regions for the detection of esophageal dysplasia/early carcinoma.

In the current study, patients were selected with esophageal symptoms and $86(10.6 \%)$ patients were observed to have dysplasia/early carcinoma, among whom 19 (2.3\%, 19/812) cases had severe dysplasia/early carcinoma. The detection rate of severe dysplasia/early carcinoma almost reached that of the high-risk population (13-17). In this study, these patients were divided into three groups according to symptoms, and $8.3 \%$ patients who had reflux, $17.2 \%$ patients who had dysphagia, and $15.8 \%$ patients who had globus sensation, were also observed to have dysplasia/early ESCC. The results indicated that increased attention must be given to the patients with esophageal symptoms, and Lugol chromoendoscopy should be recommended as a routine procedure in patients with esophageal symptoms, similarly to those high-risk patients with head and neck, or tracheobronchial squamous cell carcinomas, or had an alcohol or tobacco addiction, or lived in a high-risk region (13-17).

Previous studies have identified a positive correlation between USL size and the probability of clinically significant neoplasia $(6,18,19)$. In high-risk regions, high grade dysplasia/carcinoma is identified in $31 \%$ (46/149) patients with USLs of $<1 \mathrm{~cm}(6)$. The current study found that only $4(5.3 \%)$ patients with mild dysplasia had USLs of 0.5-1.0 cm, while 51 patients with dysplasia/early ESCC had USLs $>1.0 \mathrm{~cm}$, including 14 cases with severe dysplasia/early ESCC. The results indicated that the probability of USLs $<1.0 \mathrm{~cm}$ being severe dysplasia/early ESCC was low in low-risk regions. As it is challenging to conduct biopsies for USLs of $<0.5 \mathrm{~cm}$, and due to the current results regarding the correlation between USL size and the probability of dysplasia/early ESCC, it does not appear that biopsies are required for USLs of $<0.5 \mathrm{~cm}$ in diameter.

The Japanese Society for Esophageal Disease defined superficial esophageal carcinoma as lesions in which infiltration does not go beyond the submucosal layer, and subclassified these into the six categories: Mucosal 1, mucosal 2, mucosal 3, submucosal 1, submucosal 2 and submucosal 3 (20). It has previously been reported that mucosal 1 and mucosal 2 carcinomas have no lymph node and distal metastasis (21). In the current study, we defined early carcinoma as a tumor not beyond submucosal 2 . As esophageal severe dysplasia and early ESCC have a similar treatment and prognosis, these were grouped together.

The current study also found that it is extremely important to rinse the mucosa prior to staining and it not necessary to pretreat the mucosa with a mucolytic agent (9). The formula of the iodine solution that we used was stronger than the Lugol's solution used in other studies $(8,9)$. This formula ensured that the USLs were clearly visible for 5-9 min, allowing enough time for photographs to be recorded and biopsies to be conducted. If the stain did begin to fade prior to the biopsy, restaining was easily accomplished and effective.

In total, $20 \%$ patients experienced discomfort in this study. The predominant discomfort was a sensation of esophageal burning and pain, which appeared to be caused by gastric distention and/or reflux of iodine, and was minimized by careful suction of air and iodine from the stomach prior to the removal of the endoscope.

In conclusion, Lugol chromoendoscopy improves the detection of dysplasia/early ESCC in patients with esophageal symptoms in low-risk regions. Lugol chromoendoscopy must therefore be considered in addition to routine endoscopy in patients with esophageal symptoms.

\section{Acknowledgements}

This study was supported in part by grants from the Municipal science and technology commission of China (grant no. Z101100055610031).

\section{References}

1. Ferlay J, Shin HR, Bray F, et al: Estimates of worldwide burden of cancer in 2008: GLOBOCAN 2008. Int J Cancer 127: 2893-2917, 2010.

2. Sant M, Aareleid T, Berrino F, et al: EUROCARE-3: survival of cancer patients diagnosed 1990-94-results and commentary. Ann Oncol 14: v61-v118, 2003.

3. Jemal A, Siegel R, Ward E, Hao Y, Xu J, Murray T and Thun MJ: Cancer statistics, 2008. CA Cancer J Clin 58: 71-96, 2008.

4. Inoue $\mathrm{H}$ and Endo M: Endoscopic esophageal mucosal resection using a transparent tube. Surg Endosc 4: 189-201, 1990.

5. Wang LD, Zhou Q and Yang CS: Esophageal and gastric cardia epithelial cell proliferation in northern Chinese subjects living in a high-incidence area. J Cell Biochem Suppl 28-29: 159-165, 1997.

6. Dawsey SM, Fleischer DE, Wang GQ, et al: Mucosal iodine staining improves endoscopic visualization of squamous dysplasia and squamous cell carcinoma of the esophagus in Linxian, China. Cancer 83: 220-231, 1998.

7. Zhang SW, Zhang M, Li GL, et al: An analysis of incidence and mortality of esophageal cancer in China, 2003-2007. China Cancer 21: 241-244, 2012.

8. Shao Y, Ji M, Wu YD, et al: The clinical study of detection of dysplasia and early esophageal squamous cell carcinoma (ESCC) by using $2.5 \%$ lugol chromoendoscopy. J Clin Intern Med 23: 534-536, 2006.

9. Sugimachi K, Ohno S, Matsuda H, Mori M, Matsuoka H and Kuwano H: Clinicopathologic study of early stage esophageal carcinoma. Br J Surg 76: 759-763, 1989.

10. Shao Y, Tan W and Zhang S: P53 gene codon 72 polymorphism and risk of esophageal squamous cell carcinoma: a case-control study in a Chinese population. Dis Esophagus 21: 139-143, 2008.

11. Dawsey SM, Lewin KJ, Liu FS, Wang GQ and Shen Q: Esophageal morphology from Linxian, China: squamous histologic findings in 754 patients. Cancer 73: 2027-2037, 1994.

12. Kodama M and Kakegawa T: Treatment of superficial cancer of the esophagus: a summary of responses to a questionnaire on superficial cancer of the esophagus in Japan. Surgery 123: 432-439, 1998.

13. Kaneko K, Murakami Y,Katagiri A, et al: Does daily alcohol and/or cigarette consumption cause low-grade dysplasia, a precursor of esophageal squamous cell carcinoma? J Clin Gastroenterol 44: 173-179, 2010.

14. Boller D, Spieler P, Schoenegg R, et al: Lugol chromoendoscopy combined with brush cytology in patients at risk for esophageal squamous cell carcinoma. Surg Endosc 23: 2748-2754, 2009.

15. Dubuc J, Legoux J, Winnock M, et al: Endoscopic screening for esophageal squamous-cell carcinoma in high-risk patients: a prospective study conducted in 62 french endoscopy centers. Endoscopy 38: 690-695, 2006

16. Fukuhara T, Hiyama T, Tanaka S, et al: Characteristics of esophageal squamous cell carcinomas and lugol-voiding lesions in patients with head and neck squamous cell carcinoma. J Clin Gastroenterol 44: e27-e33, 2010.

17. Fagundes RB,deBarros SG,Pütten AC,etal:Occult dysplasia is disclosed by Lugol chromoendoscopy in alcoholics at high risk for squamous cell carcinoma of the esophagus. Endoscopy 31: 281-285, 1999.

18. Dawsey SM, Wang GQ, Weinstein WM, et al: Squamous dysplasia and early esophageal cancer in the Linxian region of China: distinctive endoscopic lesions. Gastroenterology 105: 1333-1340, 1993. 
19. Hori K, Okada H, Kawahara Y, Takenaka R, Shimizu S, et al: Lugol-voiding lesions are an important risk factor for a second primary squamous cell carcinoma in patients with esosphageal cancer or head and neck cancer. Am J Gastroenterol 106: 858-866, 2011.

20. Comprehensive Registry of Esophageal Cancer in Japan $(1998,1999)$ \& Long Term Results of Esophagectomy in Japan (1988-1997). 3rd edition. Japanese Society for Esophageal Disease, 2002.
21. Tachibana M, Yoshimura H, Kinugasa S, et al: Clinicopathological features of superficial squamous cell carcinoma of the esophagus. Am J Surg 174: 49-53, 1997. 